\title{
Artificial Intelligence Conferences Closeness
}

\author{
Sébastien Konieczny and Emmanuel Lonca \\ CRIL - CNRS, Université d'Artois, France \\ \{konieczny, lonca\}@cril.fr
}

\begin{abstract}
We study the evolution of Artificial Intelligence conference closeness, using the coscinus tool. coscinus computes the closeness between publication supports using the co-publication habits of authors: the more authors publish in two conferences, the closer these two conferences. In this paper we perform an analysis of the main Artificial Intelligence conferences based on principal components analysis and clustering performed on this closeness relation.
\end{abstract}

\section{Introduction}

It is often hard and artificial to put boundaries on scientific domains. This is especially true for Artificial Intelligence, due to the highly different theoretical techniques that are used, and the huge spectrum of applications domains.

Similarly it can be difficult to judge the proximity or closeness of different Artificial Intelligence communities, and the historical evolution of this closeness.

One possibility is to look at the contents of the main conferences of these communities, in order to analyse this kind of change. This can be done for instance by trying to analyse and to match the title of the sessions of these conferences. But this process would require (subjective) interpretations and text analysis.

A more objective way of analysing the closeness between communities is by looking at the publication habits of researchers. Starting from the hypothesis that mosts researchers are specialists of a given single domain, or of a set of connected domains, this means that the publication supports (conferences and journals) used by a researcher connect these publications supports together (around this researcher expertise). So this means that if there are many researchers that publish papers in two distinct conferences, these conferences are clearly thematically connected. So, by looking at the publication habits of researchers, one can draw a landscape of publication supports, and then obtain objective information about the communities and the domains.

This is the aim of the coscinus tool (http://www.coscinus. org), that performs such an analysis from publications data issued from DBLP (https://dblp.uni-trier.de), that is currenlty the largest and most stable database of computer science publications, with more than 4 millions of records (more than 1.5 millions of journal papers and more than 2 millions of conference and workshop papers) in 2018 (source https://dblp.org/statistics/recordsindblp.html).

In order to perform a study of the historical evolution of Artificial Intelligence conference closeness, we have adapted this tool by adding a temporal window selection, and focusing on Artificial Intelligence publication supports. This adapted tool can be find here: http://www.coscinus.org/ai. In this paper we will sum up and analyze some observations that can be made by using this tool.

In Section 2 we will describe the coscinus methodology, how the closeness measure is defined, and how it is used for the principal component analysis and for the clustering. In Section 3 we will study the closeness of the IJCAI conference with conferences from other domains, in order to have a global view of this closeness with other main computer science conferences. In Section 4 we will perform a similar analysis but focusing only on IJCAI-related AI conferences.

\section{Coscinus}

The data used in the coscinus project (http://www.coscinus. org) are issued from two sources:

- The DBLP database (https://dblp.uni-trier.de)

- The CORE ranking (http://www.core.edu.au/conference-portal)

The CORE ranking classify publication supports (conferences and journals) on several categories. In coscinus we restrict ourselves to publication supports in categories $A^{*}, A$, $B$ and $C$. Here is the meaning of the categories as summed up in http://www.core.edu.au/conference-portal:

- $A^{*}$ - flagship conference, a leading venue in a discipline area

- A - excellent conference, and highly respected in a discipline area

- B - good conference, and well regarded in a discipline area

- C - other ranked conference venues that meet minimum standards

For replicability, the data used for the experimentations described in this paper are available here: http://coscinus.org/ data. The version of the DBLP database used for experimentations described in this paper is the 22 may 2018 one. 
We use the last versions of the CORE ranking, i.e. CORE 2018 for conferences and CORE 2010 for journals. We provide also the CSV file linking the publication supports entries from DBLP and the ones from the CORE ranking that we use for coscinus and for the reported experiments. Since there is no standardized key naming of publication supports (globally based on acronyms of the support, but not always and with possible variations), that some conference names changed during their history, and that DBLP put some conferences under a same key, some publication supports are missing because of a mismatch of entry keys between DBLP and CORE.

For the data used in the coscinus project and for this paper, we have:

- 1941999 papers (CORE A*: 339691 / A: 583255 / B: 647668 / C: 371 385)

- 1172474 authors

- 925 conferences (CORE A*: 63 / A: 201 / B: 308 / C: 353)

- 480 journals (CORE $A^{*}$ : 51 / A: 112 / B: 137 / C: 180)

Now let us turn to the way we exploit these data. The fundamental idea is that researchers in a field tend to publish in the same conferences/journals. And most of these researchers have at most one or two fields of competence. Hence, it is possible to draw some connections between conferences/journals by looking at how much researchers have papers in the two conferences/journals.

So we build a matrix of number of publications of each author in each support (conference/journals). Then, we build a matrix [supports $\times$ supports], where we count the number of authors that publish in the two publication supports. To avoid too much noise we use a threshold to decide which authors are counted. It may happen that an author published once in a publication support that is not in his field. While it may be significant, there are also some chances that this publication is an "anomaly", that is not related at all with the mainstream work of this author. This phenomenon induces some noise in the data. As an example, suppose that an author has 9 papers in conference A, 7 in conference B, 4 in conference C, and 1 in conference D. While we can suppose that conferences A, $\mathrm{B}$ and $\mathrm{C}$ are close, this is a much weaker conclusion than $\mathrm{D}$ is related to these three conferences. The MC (Minimal Count) threshold $\mathrm{n}$ works like this: we add 1 in the matrix position [supportA, supportB] for each author that has published at least $n$ papers in support $A$ and $n$ papers in supportB. Choosing high values for $\mathrm{n}$ allows to focus on mosts prolific authors only, and reduces the noise produced by anomalies.

The obtained counting matrix is the basic information we use as closeness measure between publication supports. There is just still one issue: some publication supports publish much more papers than others, then this matrix is biased by these differences in the total numbers of accepted papers. In order to fix this issue, we use a normalization step. This turns the first, counting matrix, in a second, proportional matrix. After this normalization step is applied, we obtain as final information the proportion of authors of a publication support that also published in another one.

There are three possible standard normalizations, since to normalize the data [support1, support2], one can normal- ize on the total number of papers of support1 (nsupport1 $=$ [support1, support1]), or the total number of papers of support2 $($ nsupport2 $=$ [support2, support2])):

- $\min :[$ support1, support2]/ min(nsupport1, nsupport2)

- max:[support1, support2]/ max(nsupport1, nsupport2)

- $\operatorname{avg}:(\min +\max ) / 2$

Let us illustrate this process on a small part of the matrix with some AI conferences. The first matrix, the counting matrix is given on Table 1. This matrix is obtained from the real data from coscinus, using $M C=3$, i.e. each unity in a matrix cell [supportA, supportB] means that an author published at least 3 papers in supportA and supportB. So one can see that there are 1826 authors that published at least 3 papers at IJCAI. And among these ones, 935 also published at least 3 papers at AAAI, whereas only 54 published at least 3 papers at JELIA.

Then we give the corresponding min-normalized matrix on Table 2. This is one of these three matrices (that we will call nmatrix), depending of the chosen normalization, that is used as input of both the principal component analysis and the clustering methods.

We perform a PCA (Principal Component Analysis) on the nmatrix matrix. PCA is a classical statistical method used to reduce the number of dimensions of a set of data [Pearson, 1901; Jackson, 2003; Jolliffe, 2002]. We consider our matrix as indicating a profile of each publication support on a space composed of 1405 dimensions (the total number of publication supports), and we project these publication supports on the 2 dimensions space chosen by the PCA method (the aim of the PCA is to find the best choice for a reduced number of dimensions).

This result is computed from the nmatrix for different values of MC (MinCount threshold), the three normalization functions (min, max, avg), and for different levels of publication supports according to CORE ranking classification $\left(A^{*}, A^{*}+A, A^{*}+A+B, A^{*}+A+B+C\right)$. It is also possible, for example, to choose to display only $A^{*}$ or $A^{*}+A$ or $A^{*}+A+B$ publication supports when choosing the nmatrix based on $A^{*}+A+B+C$ publication supports.

We also perform clustering on the nmatrix matrix in order to identify clusters of publication supports. We propose two clustering methods :

- The first one is the k-means clustering method, that is one of the main clustering methods [Macqueen, 1967] (but it is not hierarchical, so clusters can be rather different when we increase the number of clusters).

- The second one is an agglomerative clustering method, implying that when we increase the number of cluster by one, one cluster will be splitted in two. More exactly we use the average-link clustering method [Ward, 1963; Rokach and Maimon, 2005].

The coscinus project allows to compute the results for conferences only, for journals only, and for both. From now on, in this study we will focus on conferences. We will also use the min-normalization in the following, and the k-means clustering method. 


\begin{tabular}{llllllllllllllll} 
& LPNMR & ECML & JELIA & CP & AAAI & TARK & KR & NIPS & ICML & CADE & UAI & ECAI & AAMAS & IJCAI & AIML \\
\hline LPNMR & 141 & 2 & 46 & 5 & 43 & 0 & 34 & 2 & 2 & 3 & 3 & 34 & 17 & 55 & 0 \\
\hline ECML & 2 & 516 & 3 & 5 & 94 & 0 & 2 & 86 & 125 & 4 & 26 & 44 & 27 & 93 & 2 \\
\hline JELIA & 46 & 3 & 109 & 2 & 32 & 2 & 42 & 2 & 3 & 13 & 2 & 50 & 23 & 54 & 7 \\
\hline CP & 5 & 5 & 2 & 361 & 126 & 3 & 20 & 13 & 9 & 15 & 17 & 70 & 34 & 122 & 1 \\
\hline AAAI & 43 & 94 & 32 & 126 & 2496 & 15 & 128 & 271 & 281 & 28 & 163 & 203 & 390 & 935 & 4 \\
\hline TARK & 0 & 0 & 2 & 3 & 15 & 53 & 11 & 1 & 0 & 2 & 6 & 6 & 13 & 18 & 5 \\
\hline KR & 34 & 2 & 42 & 20 & 128 & 11 & 212 & 10 & 7 & 23 & 29 & 94 & 43 & 161 & 6 \\
\hline NIPS & 2 & 86 & 2 & 13 & 271 & 1 & 10 & 1844 & 620 & 5 & 173 & 24 & 51 & 174 & 2 \\
\hline ICML & 2 & 125 & 3 & 9 & 281 & 0 & 7 & 620 & 1088 & 4 & 143 & 30 & 50 & 206 & 2 \\
\hline CADE & 3 & 4 & 13 & 15 & 28 & 2 & 23 & 5 & 4 & 343 & 2 & 28 & 8 & 56 & 8 \\
\hline UAI & 3 & 26 & 2 & 17 & 163 & 6 & 29 & 173 & 143 & 2 & 460 & 33 & 43 & 130 & 2 \\
\hline ECAI & 34 & 44 & 50 & 70 & 203 & 6 & 94 & 24 & 30 & 28 & 33 & 627 & 171 & 318 & 8 \\
\hline AAMAS & 17 & 27 & 23 & 34 & 390 & 13 & 43 & 51 & 50 & 8 & 43 & 171 & 1421 & 317 & 7 \\
\hline IJCAI & 55 & 93 & 54 & 122 & 935 & 18 & 161 & 174 & 206 & 56 & 130 & 318 & 317 & 1826 & 12 \\
\hline AIML & 0 & 2 & 7 & 1 & 4 & 5 & 6 & 2 & 2 & 8 & 2 & 8 & 7 & 12 & 46
\end{tabular}

Table 1: Counting Matrix

\begin{tabular}{|c|c|c|c|c|c|c|c|c|c|c|c|c|c|c|c|}
\hline & LPNMR & ECML & JELIA & $\mathrm{CP}$ & AAAI & TARK & $\mathrm{KR}$ & NIPS & ICML & CADE & UAI & ECAl & AAMAS & IJCAI & AIML \\
\hline LPNMR & 100 & 1.41 & 42.20 & 3.54 & 30.49 & 0 & 24.11 & 1.41 & 1.41 & 2.12 & 2.12 & 24.11 & 12.05 & 39.00 & 0 \\
\hline ECML & 1.41 & 100 & 2.75 & 1.38 & 18.21 & 0 & 0.94 & 16.66 & 24.22 & 1.16 & 5.65 & 8.52 & 5.23 & 18.02 & 4.34 \\
\hline JELIA & 42.20 & 2.75 & 100 & 1.83 & 29.35 & 3.77 & 38.53 & 1.83 & 2.75 & 11.92 & 1.83 & 45.87 & 21.10 & 49.54 & 15.21 \\
\hline $\mathrm{CP}$ & 3.54 & 1.38 & 1.83 & 100 & 34.90 & 5.66 & 9.43 & 3.60 & 2.49 & 4.37 & 4.70 & 19.39 & 9.41 & 33.79 & 2.17 \\
\hline AAAl & 30.49 & 18.21 & 29.35 & 34.90 & 100 & 28.30 & 60.37 & 14.69 & 25.82 & 8.16 & 35.43 & 32.37 & 27.44 & 51.20 & 8.69 \\
\hline TARK & 0 & 0 & 3.77 & 5.66 & 28.30 & 100 & 20.75 & 1.88 & 0 & 3.77 & 11.32 & 11.32 & 24.52 & 33.96 & 10.86 \\
\hline $\mathrm{KR}$ & 24.11 & 0.94 & 38.53 & 9.43 & 60.37 & 20.75 & 100 & 4.71 & 3.30 & 10.84 & 13.67 & 44.33 & 20.28 & 75.94 & 13.04 \\
\hline NIPS & 1.41 & 16.66 & 1.83 & 3.60 & 14.69 & 1.88 & 4.71 & 100 & 56.98 & 1.45 & 37.60 & 3.82 & 3.58 & 9.52 & 4.34 \\
\hline ICML & 1.41 & 24.22 & 2.75 & 2.49 & 25.82 & 0 & 3.30 & 56.98 & 100 & 1.16 & 31.08 & 4.78 & 4.59 & 18.93 & 4.34 \\
\hline CADE & 2.12 & 1.16 & 11.92 & 4.37 & 8.16 & 3.77 & 10.84 & 1.45 & 1.16 & 100 & 0.58 & 8.16 & 2.33 & 16.32 & 17.39 \\
\hline UAI & 2.12 & 5.65 & 1.83 & 4.70 & 35.43 & 11.32 & 13.67 & 37.60 & 31.08 & 0.58 & 100 & 7.17 & 9.34 & 28.26 & 4.34 \\
\hline ECAl & 24.11 & 8.52 & 45.87 & 19.39 & 32.37 & 11.32 & 44.33 & 3.82 & 4.78 & 8.16 & 7.17 & 100 & 27.27 & 50.71 & 17.39 \\
\hline AAMAS & 12.05 & 5.23 & 21.10 & 9.41 & 27.44 & 24.52 & 20.28 & 3.58 & 4.59 & 2.33 & 9.34 & 27.27 & 100 & 22.30 & 15.21 \\
\hline IJCAI & 39.00 & 18.02 & 49.54 & 33.79 & 51.20 & 33.96 & 75.94 & 9.52 & 18.93 & 16.32 & 28.26 & 50.71 & 22.30 & 100 & 26.08 \\
\hline AIML & 0 & 4.34 & 15.21 & 2.17 & 8.69 & 10.86 & 13.04 & 4.34 & 4.34 & 17.39 & 4.34 & 17.39 & 15.21 & 26.08 & 100 \\
\hline
\end{tabular}

Table 2: Min-normalized Matrix

Let us illustrate what kind of information and vizualisation we can obtain from the general coscinus website, before focusing on AI conferences and on the historical study.

Figure 1 shows the 2-dimensional representation obtained by PCA ( $w i t h \mathrm{MC}=3$, normalization=min), and the clusters obtained by k-means (for 12 clusters) of the $A^{*}$ conferences in the system (63 conferences).

One can note that the IJCAI cluster is composed of IJCAI, ICAPS, AAAI, AAMAS and KR. And that there is a "machine learning cluster" composed of NIPS, ICML, COLT,

\section{UAI and RSS.}

One can note also that there is a clear "Artificial Intelligence" region (these two clusters), and the other distinct region is the "Database + Web" one, that is clearly outside the main region with all other $A^{*}$ conferences.

These results are obtained by taking only the $63 A^{*}$ conferences into account. We can obtain more detailed results by considering additionally $A$ conferences. Figure 2 shows (under the same parameters) the $A+A^{*}$ conferences in the system (264 conferences). Figure 3 is a zoom on the IJCAI area, that 


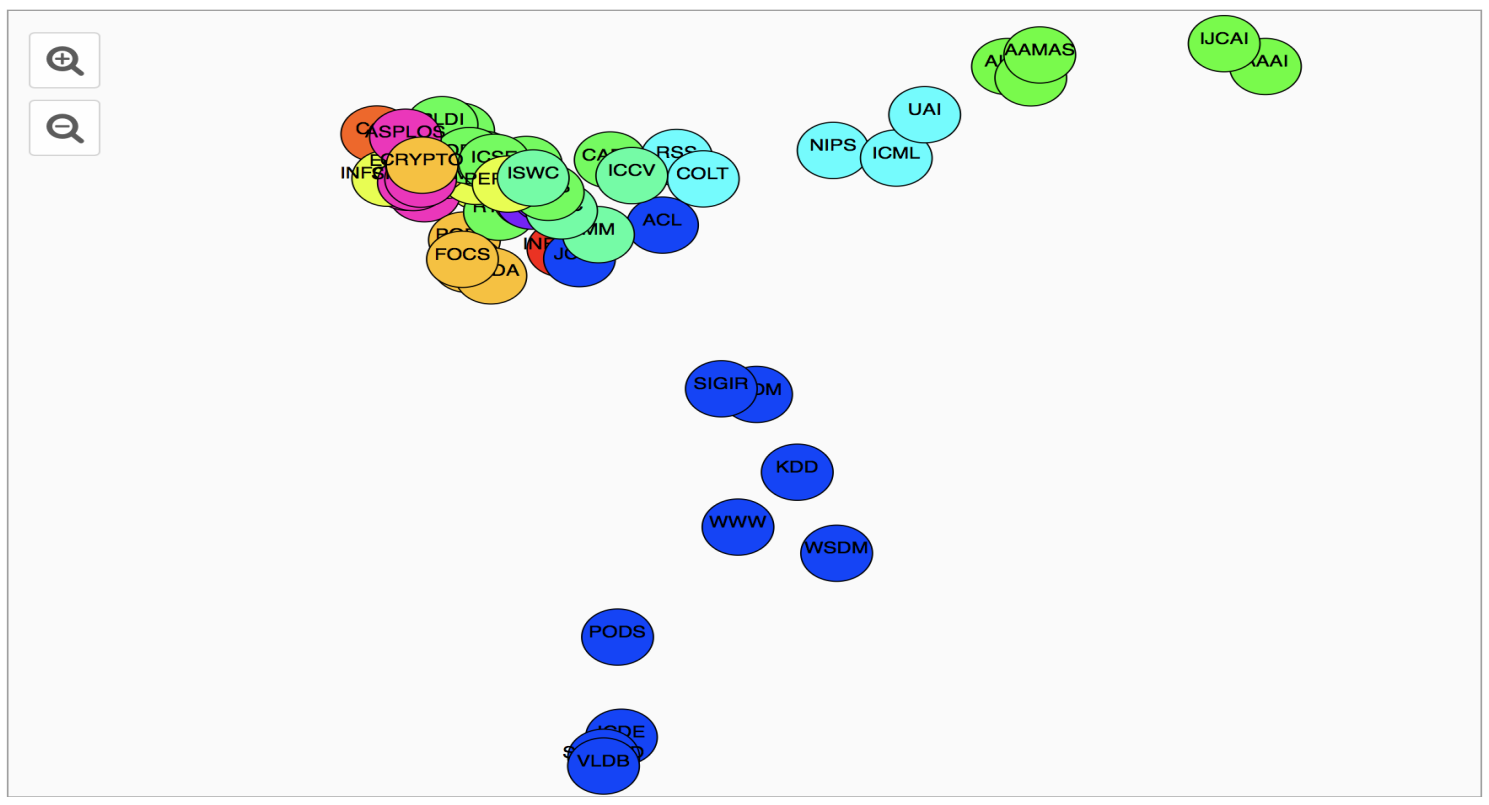

Figure 1: $A^{*}$ conferences $(\mathrm{MC}=3$, norm $=\min , 12$ clusters $)$

allows to have a better vizualization of the AI related part.

In this case the IJCAI cluster (still for 12 clusters for the $\mathrm{k}$-means method) is composed of IJCAI, LPNMR, PKDD, JELIA, AISTATS, ICAPS, ICDM, AAAI, KDD, KR, NIPS, ICML, UAI, ECAI, CIKM, KCAP, WSDM, WWW, PAKDD, IDA, SIGIR, SDM, CVPR, So we obtain an unsurprising result, with, besides purely AI conferences, conferences on data-mining, web, and vision.

These results are obtained through the whole set of DBLP data. One interesting question is to study the historical evolution of conference closeness. This is what we address in the following (in order to have a better vizualization and more information, the reader can go to http://www.coscinus.org/ai).

\section{IJCAI Closest A* Conferences}

We will use a temporal window selection in order to study the historical evolution of closeness between conferences. We have chosen a 5 years window in order to have at least 2 editions of biennial conferences. We have centered the windows on the central year. So in the following when we will refer for instance to 2015, this means the 2013-2017 window period.

With a small temporal selection we can not use a big value for MC (Minimal Count). In the standard case (without temporal selection) we use $\mathrm{MC}=3$ as default. Here, with 5year windows, we select $\mathrm{MC}=1$ in order to obtain sufficiently many data. One can consider that on a small temporal window the "anomalies" (i.e. a researcher publishing a paper in a conference outside her expertise domain) will not be numerous, so that the introduced noise will be small.

Let us illustrate in Table 3 the closeness between IJCAI and other $A^{*}$ conferences, by looking at the conferences that are in the same cluster as IJCAI for 5, 10, 20 and 30 clusters.

For 20 and 30 clusters the conferences that appear the more often are AAAI and $\mathrm{KR}$, and the ones that appear more than once are AAAI, KR, ICAPS, AAMAS and UAI, so they can be considered as the closest conferences from IJCAI. But one can not really see a temporal evolution on this set (still it is possible to notice that in 2015 LICS and ISSAC appear with 20 clusters).

For 10 clusters, one can see mostly AI-related conferences. This means that there is clear boundary of the contours of AI. There are often discussions about what is AI, with a common sentence saying that "AI is everywhere", i.e. that AI does not exist per se, and that it is closely intricated with other communities. But this shows that, as a publishing community, there is a clear contour of AI. This singularity can also be seen simply on the PCA result, since the AI conferences are grouped in one of the branches of the obtained representation.

For 5 and 10 clusters we can see conferences from other communities. In 1980 this is quite diverse, since appear a conference on information systems (ICIS), one of theoretical computer science (ISSAC) and one of cryptography (EUROCRYPT). In 1985 the only non-AI conferences are theoretical computer science conferences (LICS, POPL) and a distributed computing one (PODC). This can be maybe related to the developments of multi-agent systems, and of object/actor languages. In 1990 appear a verification conference (CAV) and an information retrieval one (SIGIR). In 1995 there are visualisation/vision/interface conferences (INFOVIS, ICCV, CHI, ISWC). Then from 2000 one can observe the convergence towards data mining and web conferences (KDD, SIGIR, WWW, WSDM), that lasts until now.

\section{IJCAI-related AI Conferences}

In this section we focus on a selection of AI conferences. We have chosen a set of $A^{*}$ and $A$ conferences from the CORE ranking. The 33 conferences we focus on are the following ones: AIML, FUZZIEEE, NIPS, ICAPS, PKDD, AAAI, IJ- 


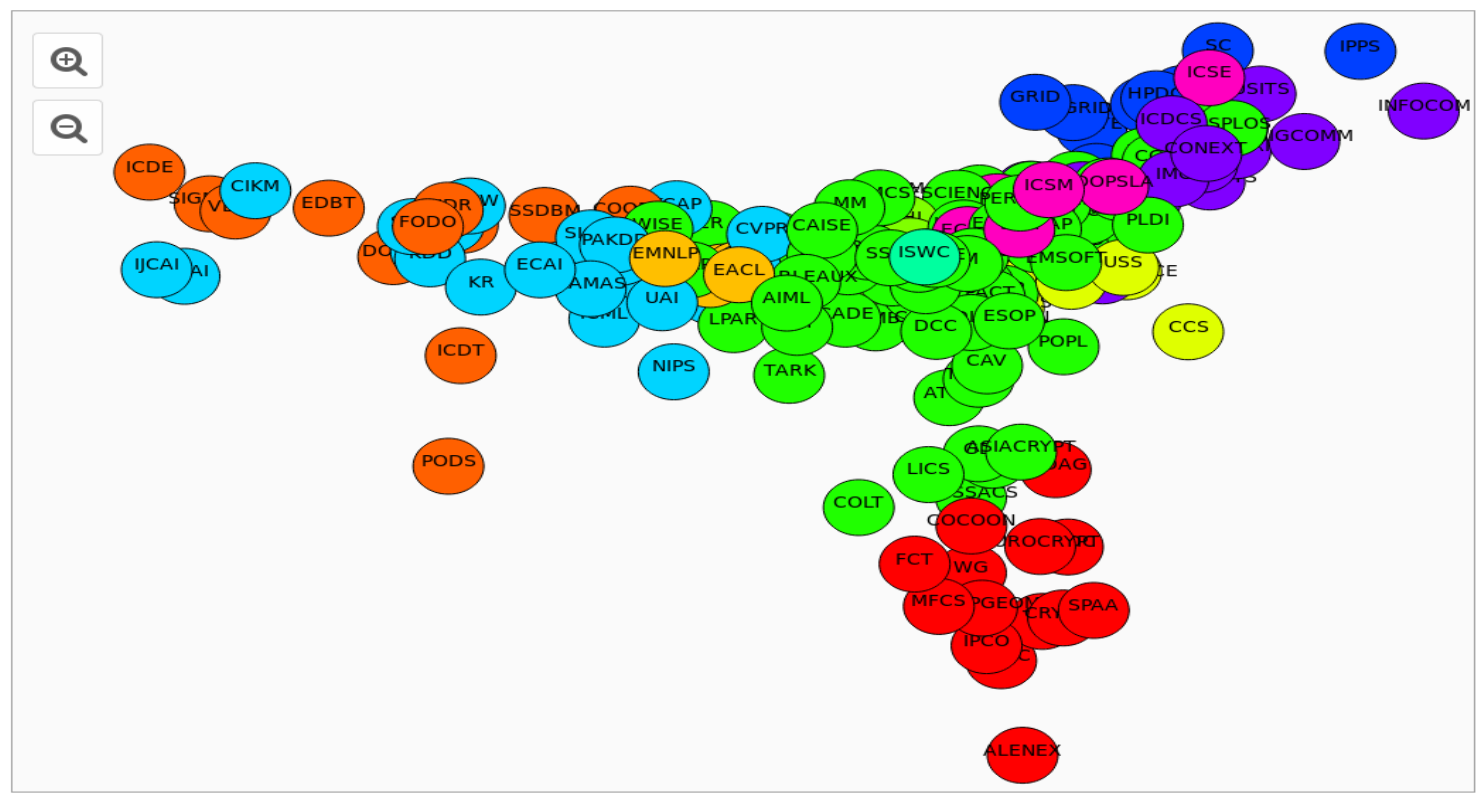

Figure 2: $A+A^{*}$ conferences $(M C=3$, norm $=\min , 12$ clusters)

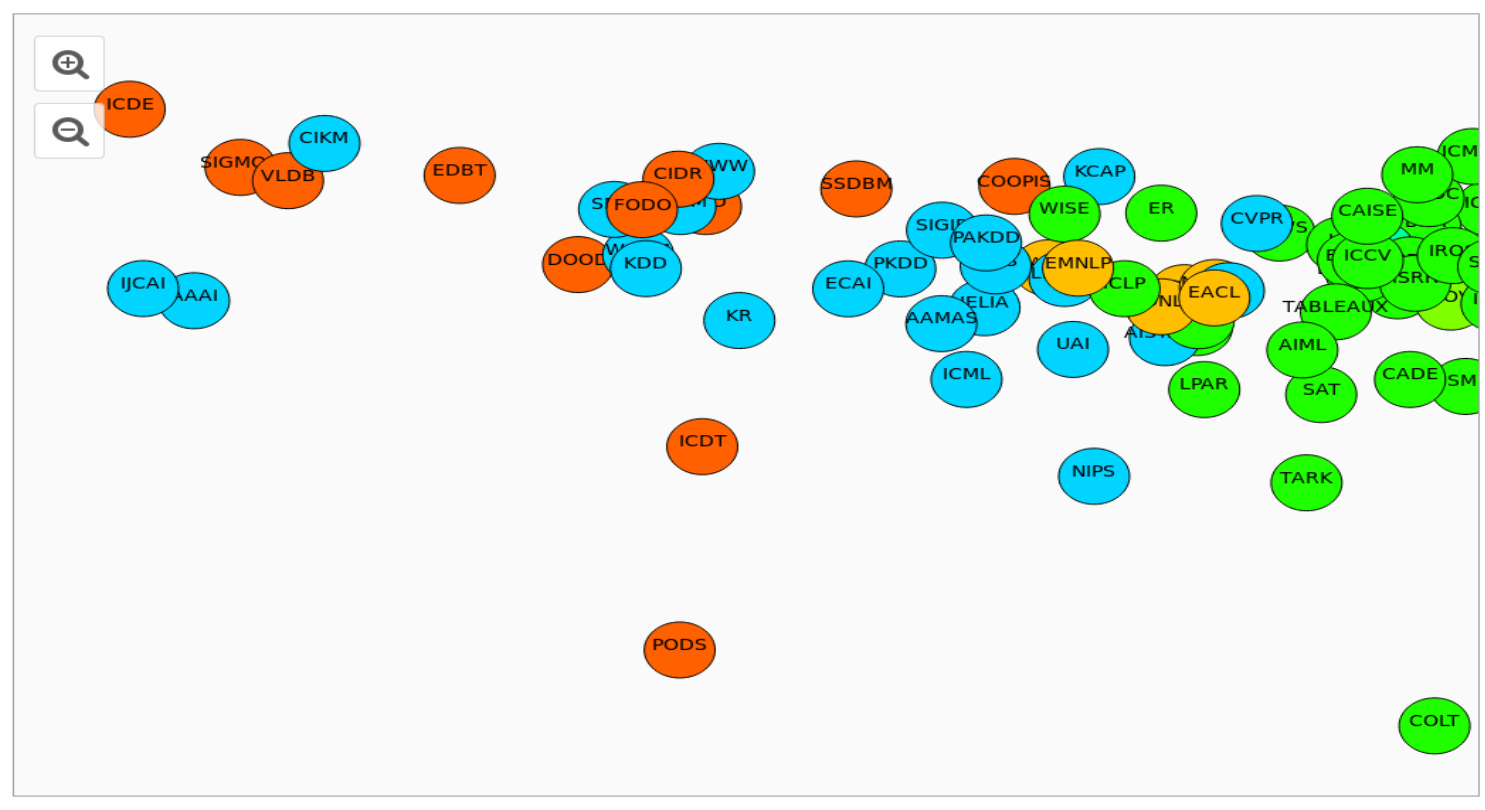

Figure 3: $A+A^{*}$ conferences - IJCAI area

CAI, UAI, ICONIP, ECAI, EMNLP, JELIA, TARK, ACL, TABLEAUX, CIKM, AISTATS, ICLP, ICDM, RSS, ICML, LPAR, ISRR, CP, KR, AAMAS, ESWS, IJCNN, CADE, COLT, SAT, LPNMR, FOGA.

The idea here is to try to identify AI sub-communities and their historical evolution. So in Table 4 we put all the clusters of these 33 conferences, when using the k-means clustering methods with 20 clusters and 30 clusters. This cluster computation is made on all computer science $A^{*}$ and $A$ conferences (that represent 239 conferences), and then we only focus on these 33 conferences and their clusters. This explains why we have less than 20 or 30 clusters in the 20/30 clusters column, since some clusters do not contain any of these 33 conferences.

A global comment on these results is that they illustrate the real singularity of artificial intelligence in the computer science community, since the clusters containing main AI conferences are mostly composed of AI conferences.

Let us first try to find conferences that are most of the times in a same cluster, that would suggest stable sub-communities. 
Proceedings of the Twenty-Seventh International Joint Conference on Artificial Intelligence (IJCAI-18)

\begin{tabular}{|c|c|c|c|c|}
\hline year $( \pm 2)$ & 5 clusters & 10 clusters & 20 clusters & 30 clusters \\
\hline 2015 & $\begin{array}{l}\text { ACL WSDM ICAPS ICDM AAAI } \\
\text { WWW KDD SIGIR KR JCDL MM } \\
\text { UAI ICCV AAMAS }\end{array}$ & ICAPS AAAI KR AAMAS & $\begin{array}{l}\text { ISSAC KR CADE } \\
\text { LICS }\end{array}$ & $\begin{array}{l}\text { ICAPS } \\
\text { AAAI KR } \\
\text { AAMAS }\end{array}$ \\
\hline 2010 & $\begin{array}{l}\text { ACL WSDM ICAPS ICDM AAAI } \\
\text { WWW KDD SIGIR KR NIPS ICML } \\
\text { COLT UAI AAMAS }\end{array}$ & $\begin{array}{l}\text { ACL WSDM ICAPS ICDM AAAI } \\
\text { WWW KDD SIGIR KR FOGA NIPS } \\
\text { ICML COLT UAI AAMAS }\end{array}$ & ICAPS AAAI & AAAI KR \\
\hline 2005 & $\begin{array}{l}\text { ICAPS AAAI KDD KR NIPS ICML } \\
\text { COLT UAI }\end{array}$ & $\begin{array}{l}\text { RSS ICAPS AAAI KR NIPS ICML } \\
\text { COLT UAI ICCV AAMAS }\end{array}$ & AAAI KR & $\begin{array}{l}\text { ICAPS } \\
\text { UAI }\end{array}$ \\
\hline 2000 & $\begin{array}{l}\text { ICAPS AAAI KDD SIGIR KR NIPS } \\
\text { ICML COLT UAI }\end{array}$ & $\begin{array}{l}\text { RTSS CAV ICDM AAAI WWW KR } \\
\text { ICML CADE MM UAI AAMAS }\end{array}$ & $\begin{array}{l}\text { ICAPS AAAI UAI } \\
\text { AAMAS }\end{array}$ & \\
\hline 1995 & $\begin{array}{l}\text { ACL KR CHI INFOVIS CADE ICCV } \\
\text { AAMAS ISWC }\end{array}$ & $\begin{array}{l}\text { ICAPS AAAI KDD KR FOGA NIPS } \\
\text { ICML COLT UAI }\end{array}$ & $\begin{array}{l}\text { ICAPS AAAI KR } \\
\text { AAMAS }\end{array}$ & KR \\
\hline 1990 & $\begin{array}{l}\text { ACL CAV ICAPS AAAI SIGIR KR } \\
\text { ICML CADE COLT UAI }\end{array}$ & ACL ICML CADE UAI & AAAI KR ICML & AAAI KR \\
\hline 1985 & ACL CADE LICS & $\begin{array}{l}\text { ACL PODC POPL AAAI STOC FOCS } \\
\text { CADE LICS }\end{array}$ & & \\
\hline 1980 & $\begin{array}{l}\text { AAAI ISSAC ICIS CADE EURO- } \\
\text { CRYPT }\end{array}$ & ACL & AAAI & \\
\hline
\end{tabular}

Table 3: $A^{*}$ conferences in the same cluster than IJCAI (k-means clustering)

We can identify a first big group composed of AAAI, IJCAI, ECAI, KR and AAMAS. Then one can also identify a machine learning group (NIPS, COLT, ICML, AISTATS, UAI), a natural language one (ACL, EMNLP), an automated reasoning one (LPAR, CADE, TABLEAUX, LPNMR, SAT), and lastly a data-mining one (PKDD, CIKM, ICDM), but that seems less strongly connected than the other ones.

One can note that the conferences on neural networks IJCNN and ICONIP are not closely connected to NIPS.

One can note also that the robotics conference group (RSS, ISRR) is often connected to machine learning and neural networks conferences, but neither to the planning and scheduling one (AIPS).

It is also interesting to note that on the 30 clusters column, ICLP, CP and JELIA appear sometimes with the IJCAI group (AAAI, IJCAI, ECAI, KR, AAMAS), and sometimes in the automated reasoning group (LPAR, CADE, TABLEAUX, LPNMR, SAT), so this can be interpreted as being at the articulation between these two sub-communities (and this articulation is clear on the 20 clusters columns, where these two groups are usually unioned in a single one). It could be interesting to look at if some scientific thematic changes could explain the transition to one group to the other.

We do not have space to put here the corresponding landscapes obtained with PCA, but the reader can go on http: //www.coscinus.org/ai to explore them.

\section{Conclusion}

We described the coscinus project, that allows to exploit DBLP data in order to compute proximity between publication supports, and that allows to study the contours of computer science communities. For this paper we added a temporal window selection feature and the possibility to focus on AI conferences only. We think that it can prove useful in order to study the evolution of computer science in general, and of artificial intelligence in particular. We made a very basic use of this tool in this paper, but we believe that it could prove much more useful if combined with other observations.

\section{Acknowledgments}

This work has been partly supported by the project CPER DATA from the "Hauts-de-France" Region.

\section{References}

[Jackson, 2003] J. Edward Jackson. A User's Guide to Principal Components. Wiley Series in Probability and Statistics. Wiley-Interscience, 2003.

[Jolliffe, 2002] Ian Jolliffe. Principal component analysis. Springer Verlag, 2002.

[Macqueen, 1967] James Macqueen. Some methods for classification and analysis of multivariate observations. In In 5-th Berkeley Symposium on Mathematical Statistics and Probability, pages 281-297, 1967.

[Pearson, 1901] Karl Pearson. On lines and planes of closest fit to systems of points in space. Philosophical Magazine, 2(6):559-572, 1901.

[Rokach and Maimon, 2005] Lior Rokach and Oded Maimon. Clustering methods. In Oded Maimon and Lior Rokach, editors, The Data Mining and Knowledge Discovery Handbook., pages 321-352. Springer, 2005.

[Ward, 1963] Joe H. Ward. Hierarchical grouping to optimize an objective function. Journal of the American Statistical Association, 58(301):236-244, 1963. 


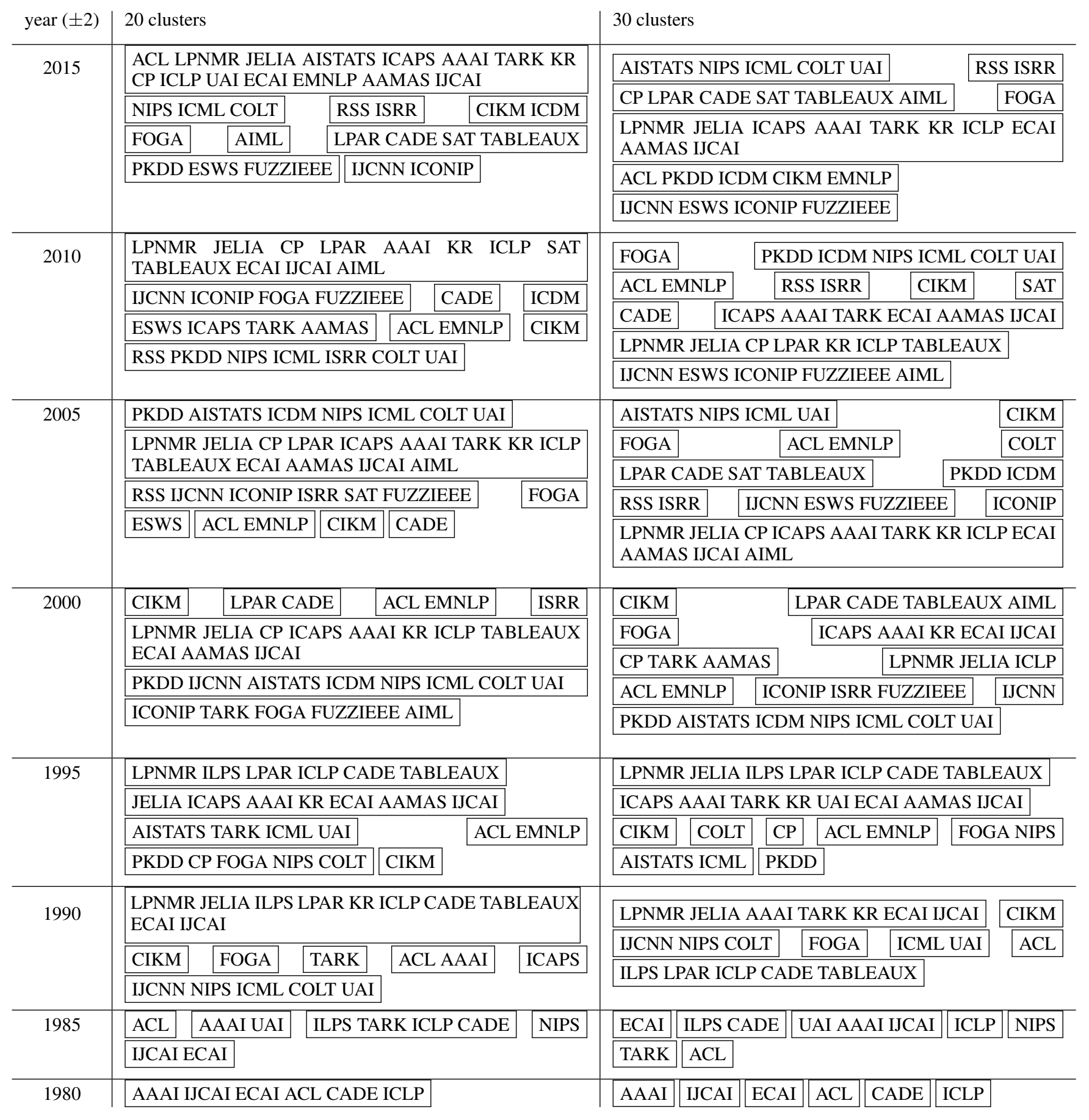

Table 4: Clusters of $A$ and $A * A I$ conferences (k-means clustering) 